\title{
CFD modeling of airflows and contaminant transport in an aircraft cabin
}

\author{
Jiuzhou Zhang ${ }^{a}$, Yunze Wang ${ }^{a}$, Z.F. Tiana, Tien-Fu Lu', Mohamed Awadalla \\ ${ }^{a}$ The School of Mechanical Engineering, The University of Adelaide, South Australia, SA 5005, Australia \\ Email: zhao.tian@adelaide.edu.au
}

\begin{abstract}
There are an increasing number of passengers undertaking air travels on commercial airliners throughout the world annually. During the flights, passengers are possibly exposed to different contaminants such as bacterial and $\mathrm{CO}_{2}$ from other passengers. As airliner cabins have high occupant density and flights can last from 1 to 20 hours, transport of contaminant could have serious impact on both passengers and aircraft crew. It is important to understand airflows and contaminant transport inside the aircraft cabin in order to reduce the negative impacts. Current aircraft cabin airflows can be analyzed by two different methods, experimental measurements and computer modeling. With the rapid increase in computer power, computer modeling is becoming more popular in study of aircraft cabin flows. Computational fluid dynamics (CFD) is the most used modeling approach since it is relatively inexpensive, fixable and able to obtain high level resolution results.
\end{abstract}

The main scope of this research is to develop a frame work to simulate airflows and trace contaminant transport in an aircraft cabin using CFD. The predicted airflows and contaminant concentration are then used to train an Artificial Intelligent (AI) system. This trained AI system will be able to trace back the possible source of the contaminant once the transmission of contaminant happens in the aircraft cabin, e.g. the severe acute respiratory syndrome (SARS) transmission in a flight in Hong Kong in 2003.

This paper reports the development of the CFD model of aircraft cabin flows and the transport of SARS in the cabin. In the project, the first milestone is to produce a section of an aircraft cabin of Airbus 320 using ANSYS/Design-Modeller. The cabin model includes half of the cabin with 7 rows of seats. The second milestone is to mesh the geometry using ANSYS/Meshing. The third milestone is to set up boundary conditions for both airflows and contaminant in ANSYS/CFX. The final objective is to solve the solutions in CFD and transfer the CFD results to an AI system developed by the authors.

Some CFD predictions of the airflow patterns and contaminant transport in the cabin are reported in the paper. It is found that the flow in the cabin is quit complex. There is a weak longitudinal flow that plays a significant role in the spread of contaminant in the cabin. Some preliminary results of the AI system are also presented in the paper.

Keywords: $\quad C F D$, contaminants, aircraft cabin, artificial intelligence 


\section{INTRODUCTION}

Air flows and contaminant transport in an aircraft cabins can be analysed by two approaches: experimental approach and computer modelling. With rapid increased computer power, computer modelling has become more popular in study of aircraft cabin flows and contaminant transport.

One of the modelling methods is computational fluid dynamics (CFD). CFD is very attractive in modelling airflows in aircraft cabins as it is relatively inexpensive, fixable when changing flow boundary conditions and can produce results with high resolution levels. Some of CFD studies of airflows and contaminant transport in aircraft cabins are reviewed herein. Aboosaidi et al. (1991) simulated airflows in an empty aircraft cabin model and validated their CFD results against measurements obtained by hot-wire anemometers, thermocouples and smoke visualization in actual aircraft cabins. Their CFD results qualitatively agree with the measurements. Mizuno (1992) numerically and experimentally studies airflows and contaminant transport in an aircraft cabin. Good agreement has been achieved between CFD and experimental analysis. These early investigations provide information only for empty cabins without passengers. However, it is critical to consider the impact of passengers on the airflow patterns as well as the concentration of contaminants in aircraft cabins. Airflow patterns in an aircraft cabin with heated cylinders as passengers on the seats were simulated by Singh et al. (2002). The results showed that there can be significant difference between occupied and unoccupied cabins.

Recently, there have been studies of airflows patterns and contaminant transport in occupied aircraft cabins by using CFD modeling, e.g. a one-dimensional analytical model for airborne contaminant transport in an airliner cabin developed by Mazumdar and Chen (2009). However, sometimes it is necessary to be able to trace back the possible source of the contaminant once the transmission of contaminant happens in the aircraft cabin, e.g. the Severe acute respiratory syndrome (SARS) transmission in a flight in Hong Kong in 2003 (Tsang et al., 2003). Therefore, the objective of this research is to develop a frame work to simulate airflows and trace contaminant transport in an aircraft cabin using CFD. The predicted airflows and contaminant concentration are then used to train an Artificial Intelligent (AI) system. Artificial intelligence, sometimes called machine intelligence, is a system created by the computer and programming logic. In the scientific field, AI is a study which understands how humans think and act and achieve them by ordinary computer intelligent (Russel and Norvig, 2003). The system has been developed which is able to trace and localize the pollutant resource from the information of infected cases.

\section{MODEL DESCRIPTION}

A CFD model of a section of an airbus A320-232 cabin was generated by using ANSYS/Design Modeller 14.0 based on available data (Airbus, 2011). The model $(520 \mathrm{~cm}$ long) consists 7 rows of passengers along one sides of the cabin. The passengers were modelled on a 50th percentile adult human male (U.S. Department of Transportation, 1996) and the seat was based on Airbus A320-232 economy seat geometry. All surfaces were considered smooth without slip and this setup was solved under steady condition.

\subsection{MESH}

The mesh of the aircraft cabin (Figure 1) was generated by using ANSYS Meshing. Some details of the mesh are given in Table 1 .
Table 1. Mesh statistics of the model

\begin{tabular}{|l|l|}
\hline Mesh Statistics & \\
\hline Aspect Ratio (Min, Max, Average) & $1.169,284.35,2.121$ \\
\hline Skewness (Min, Max, Average) & $8.843 \mathrm{e}-4,0.9986,0.3186$ \\
\hline Orthogonal Quality (Min, Max, Average) & $1.204,0.997,0.805$ \\
\hline Element Quality (Min, Max, Average) & $2.174 \mathrm{e}-3,0.9995,0.7646$ \\
\hline Total number of Nodes & 167416 \\
\hline Total number of Elements & 858944 \\
\hline
\end{tabular}




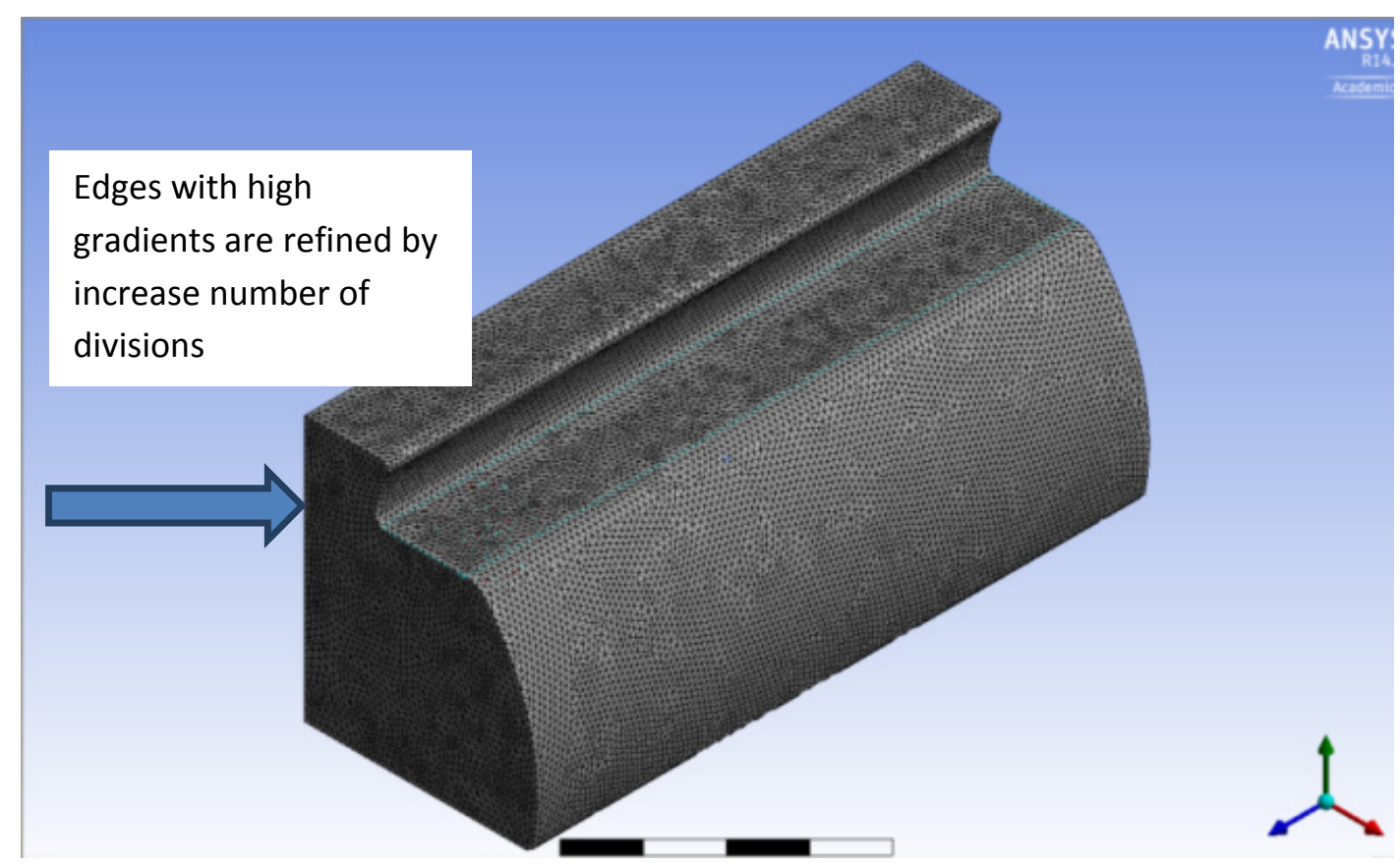

Figure 1. Isometric view of the overall mesh results for the cabin model

\subsection{MODEL SETUP}

Figure 2 and 3 below show the setup and boundary conditions of the model in CFX setup, some of the important data are listed in Table 2.

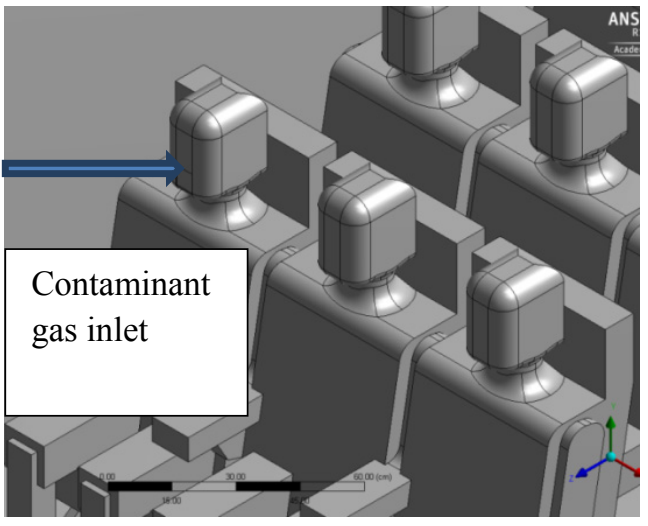

Figure 2. Aircraft cabin contaminant setup

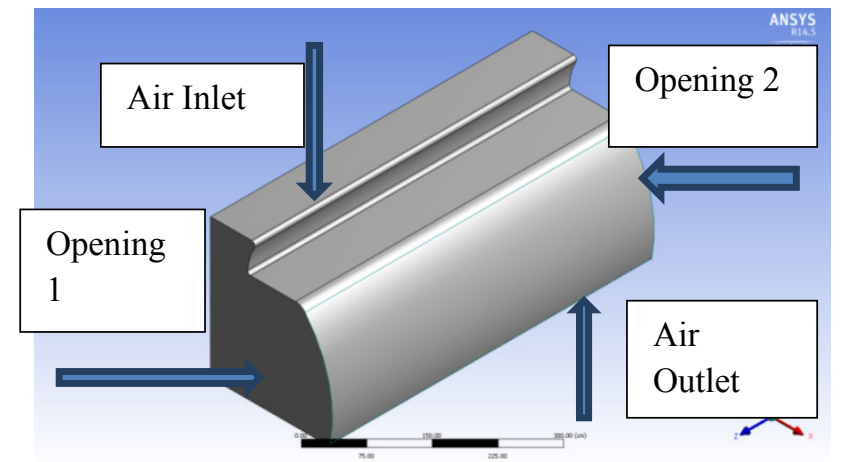

Figure 3. Aircraft cabin setup

The supply air inlet speed is $2.6 \mathrm{~m} / \mathrm{s}$ (Rai and Chen, 2012). According to Mamzudar and Chen (2009), there is a small velocity along the longitudinal direction across the aircraft cabin and it can be calculated by the equation (1):

$$
\gamma=\frac{\text { Longitudinal mass flow rate }}{\text { Mass flow rate per row }}=0.1
$$

Where, the airflow rate for the whole domain is $1.61 \mathrm{~kg} / \mathrm{s}$. Therefore, there is $0.23 \mathrm{~kg} / \mathrm{s}$ of airflow per row. Hence, longitudinal mass flow rate $=0.1 \times 0.23=0.023 \mathrm{~kg} / \mathrm{s}$. Since air density is $1.225 \mathrm{~kg} / \mathrm{m}^{3}$ and the cross section area of the model is $3.55 \mathrm{~m}^{2}$, the longitudinal velocity is $5.3 \times 10^{-3} \mathrm{~m} / \mathrm{s}$.

The contaminant SARs in aircraft cabins was simulated as a gas component. According to Schaecher (2008), the density of SARS protein is around $1200 \mathrm{~kg} / \mathrm{m}^{3}$ and the molecular weight is around $37000 \mathrm{~g} / \mathrm{mol}$. 
According to Mazumdar \& Chen (2009), the contaminants released at a flow rate of $1 \times 10^{-6} \mathrm{~kg} / \mathrm{s}$ from one passenger (shown in Figure 2).

Table 2. Model setup

\begin{tabular}{|l|l|}
\hline Model setup and boundary conditions & \\
\hline Supply air inlet speed (m/s) & 2.6 \\
\hline Turbulence Intensity (\%) & 10 \\
\hline Outlet pressure (atm) & 1 \\
\hline Opening 2 pressure (atm) & 1 \\
\hline Contaminant type & SARS \\
\hline SARs contaminant mass flow rate $(\mathrm{kg} / \mathrm{s})$ & $1 * 10-6$ \\
\hline Supply air speed at longitudinal direction for opening $1(\mathrm{~m} / \mathrm{s})$ & 0.053 \\
\hline
\end{tabular}

\section{RESULTS AND DISCUSSIONS}

Air enters the aircraft cabin at the top of the luggage cabin as shown in Figure 4 and flows downward. A large fraction of air flows out through outlets at the bottom of the right side wall. A small fraction of airs remains in the cabin and creates some circulated loops above the passengers on left side as shown in Figure 4. As shown in Figure 5, air flows in longitudinal direction from the front boundary (opening 1) to the rear boundary (opening 2). Air recirculates are found at each row in front of passengers.

Figure 6 and 7 show the concentration of SARs in the cabin. In this case, one passenger is sitting in the middle of the first row breaths out gas with SARs. The initial concentration of SARs is highest around the passenger and it spreads out to other passengers in the back row. As results of air flow along the longitudinal direction, more than one passenger exposed to the high concentration of SARs has high risk to be infected. Though the longitudinal flow in the cabin is weak compared to the local flows from the inlets above the luggage cabin, it still plays a significant role in the spread of the contaminant in the cabin in different rows. 


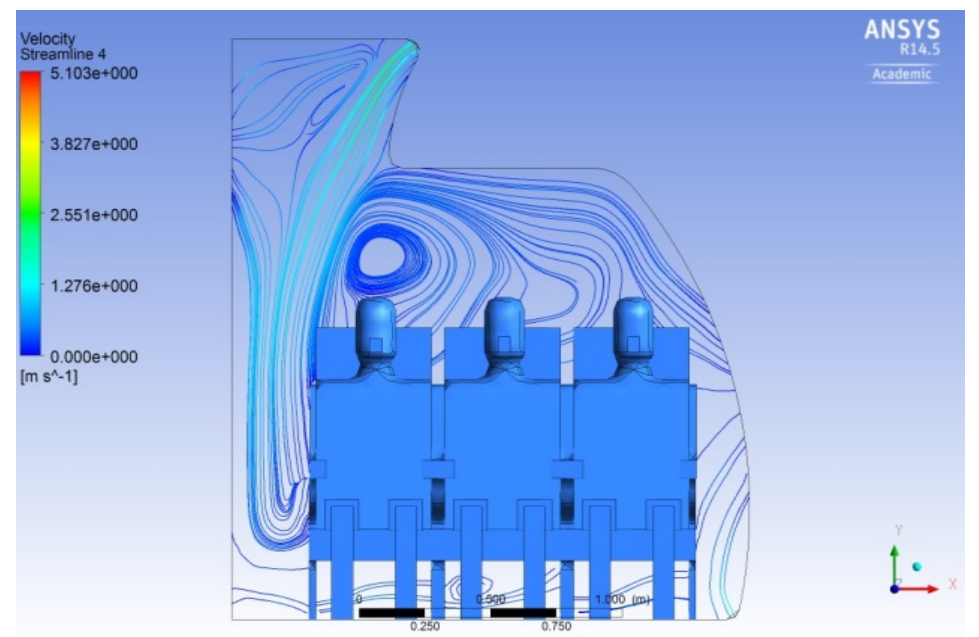

Figure 1. Cross-plane velocity streamlines profile

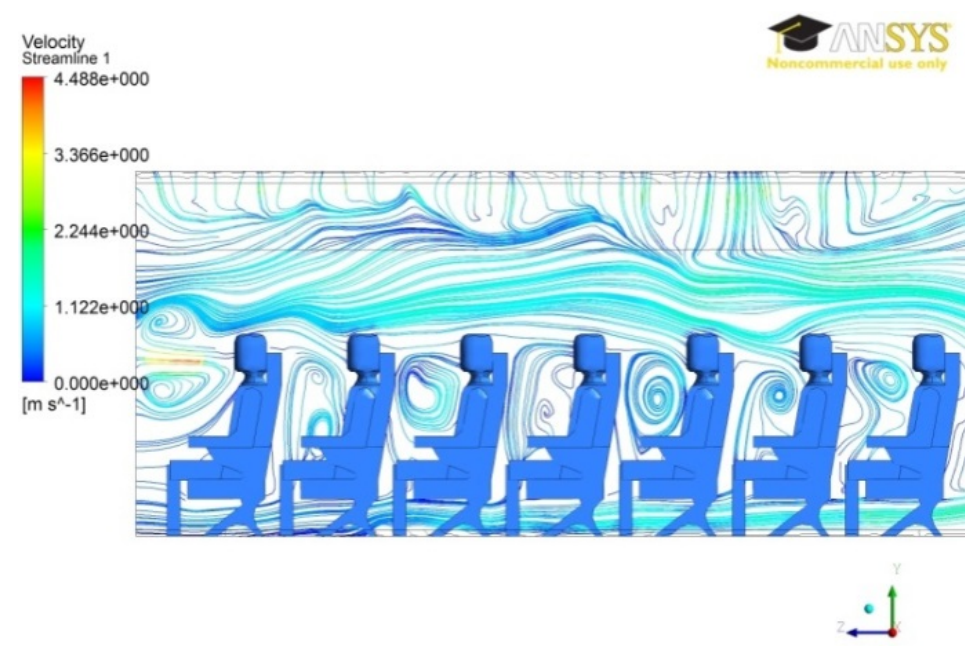

Figure 5. Side view of velocity streamlines profile

Sars.Mass Fraction Contour 1

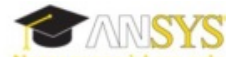

$$
\begin{array}{r}
4.954 \mathrm{e}-003 \\
4.679 \mathrm{e}-003 \\
4.404 \mathrm{e}-003 \\
4.128 \mathrm{e}-003 \\
3.853 \mathrm{e}-003 \\
3.578 \mathrm{e}-003 \\
3.303 \mathrm{e}-003 \\
3.028 \mathrm{e}-003 \\
2.752 \mathrm{e}-003 \\
2.477 \mathrm{e}-003 \\
2.202 \mathrm{e}-003 \\
1.927 \mathrm{e}-003 \\
1.651 \mathrm{e}-003 \\
1.376 \mathrm{e}-003 \\
1.101 \mathrm{e}-003 \\
8.257 \mathrm{e}-004 \\
5.505 \mathrm{e}-004 \\
2.752 \mathrm{e}-004 \\
0.000 \mathrm{e}+000
\end{array}
$$
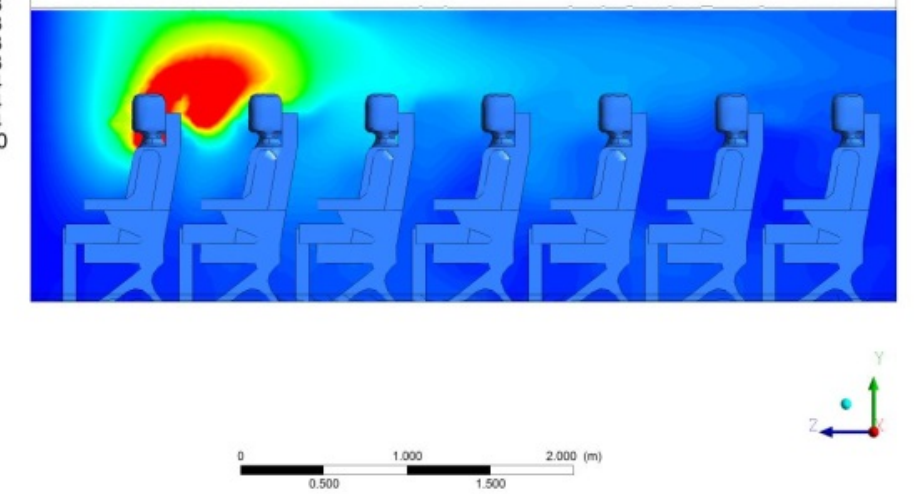

Figure 6. Side view of contaminant concentration profile 


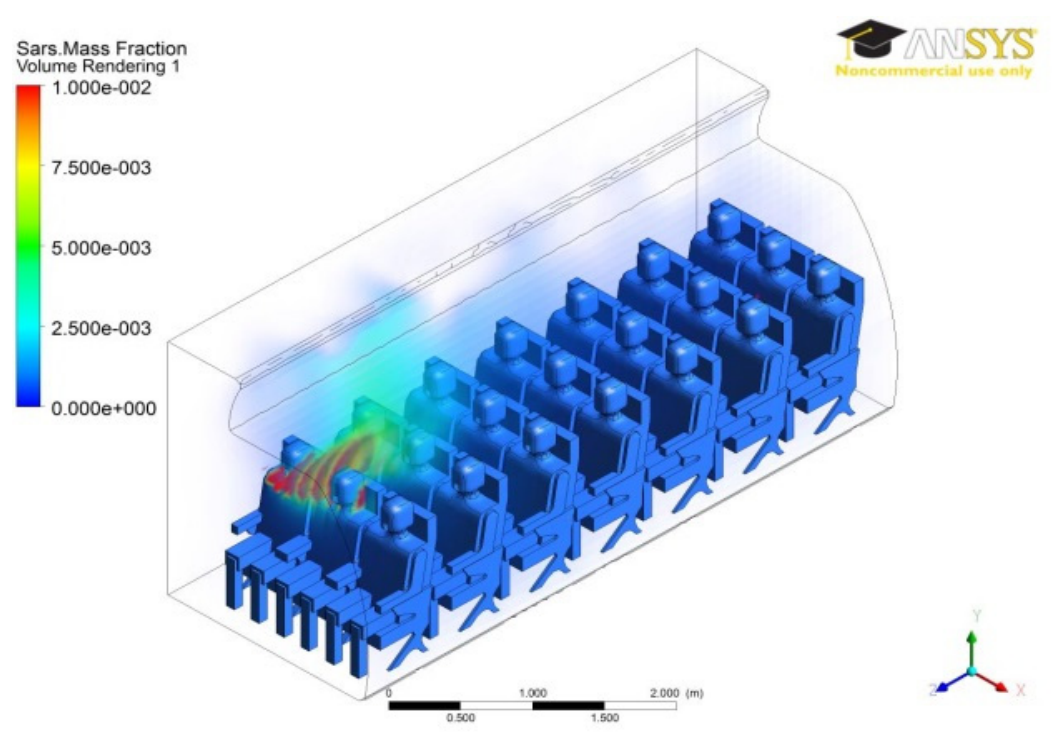

Figure 7. 3D view of contaminant concentration profile

The predicted airflow velocities and contaminant concentration are then transported into an AI system that is developed using Matlab. Artificial Intelligence is applied in this project in order to perform the tracing logic repeatedly with high efficiency. The brief explanation of this system is that it goes through all CFD nodes in the data and scale them respect to a pre-determined rule. The scaled nodes are then used to predict the pollutant resource position.

The scales are accumulated and the nodes with the highest final scale are considered as pollutant resource. Therefore a scaling system is essential and the key factors need to be determined.

The scaling algorithm uses the concept of fuzzy logic which gives a range of possibility instead of true/false values. The detailed weighting of each factor needs to be guessed based on the properties of contaminant. The primary system then iterates through several sample tests (having known solutions) and the weighting is refined according to the accuracy.

Currently, the software which is able to localize and trace the pollutant source position is under development. The basic structure and user interface has been established. However, there

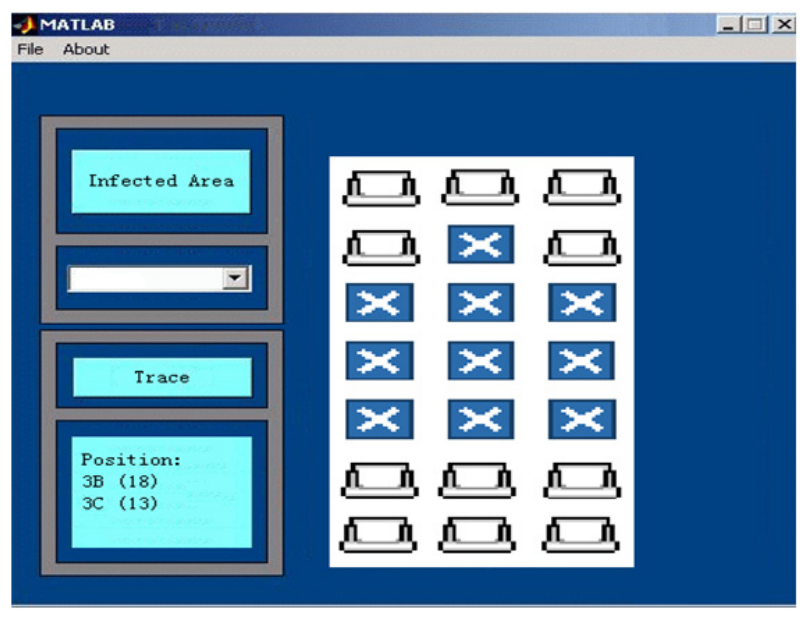

Figure 8. AI tracing user interface are limited computer resources in this research.

Therefore, the software can only deal with single contaminant source. The air flow is assumed as steady state and the analysed area is limited as 7 rows and 3 columns. Figure 8 shows the user interface. Users can manually select the infected passenger on right-side window. After clicking the 'trace' button, a list of contaminant source position would be generated. In this case, as shown in the left bottom of the window, 3B and $3 \mathrm{C}$ are the possible source positions.

\section{CONCLUSION}

This project has successfully simulated the airflows and contaminant concentration in a 7-row A320-232 aircraft cabin with a single contaminant source. The contaminant follows the flow pattern in the cabin and spreads into all 7 rows with higher concentration near the source. It is found that though the longitudinal flow in the cabin is weak compared to the flows from the inlets above the luggage cabin, it still plays a significant role in the spread of the contaminant in the cabin in different rows. The simulated airflow patterns and contaminant concentration will be used into contaminant source tracing by employing an AI system. Currently, the AI system is underdeveloping. 
Zhang et al., CFD modeling of airflows and contaminant transport in an aircraft cabin

\section{REFERENCES}

Aboosaidi, F., Warfield, M.J., and Choudhury, D. (1991). Computational fluid dynamics applications in airplane cabin ventilation system design. Proceedings - Society of Automotive Engineer, 246, 249-258.

Airbus, (2011). Airbus A320 AC Manual (Airplane Characteristics) 2-5-0, 3-4.

Jacobs, P. and Gis, W.F.D. (2006). Individual and collective climate control in aircraft cabins, International Journal of Vehicle Design, 42(1/2), 57-66.

Mazumdar, S. and Chen, Q. (2009). A one-dimensional analytical model for airborne contaminant transport in airliner cabins, Indoor Air, 19(1), 3-13.

Mizuno, T., Warfield, M.J. (1992). Development of three-dimensional thermal airflow analysis computer program and verification test. ASHIRAE Transactions, 98(2), 329-338.

Rai, A.C., and Chen, Q. (2012). Simulations of ozone distributions in an aircraft cabin using comutational fluid dynamics, Atmospheric Environment, 54, 348-357.

Russell, S., and Norving, P. (2003). Artificial Intelligene: A Modern Approach(2nd Ed.), Upper Saddle River, New Jersey, Prentice Hall.

Schaecher, S.R. (2008). Molecular Biology of The Severe Acute Respiratory Syndrome Cornavirus (SARS$\mathrm{CoV})$ Accessory Proteins ORF7a and ORF7b. Washington University, 2, 67.

Singh, A., Hosni, M.H. and Horstman, R.H. (2002). Numberical simulation of airflow in an aircraft cabin section. ASHRAE Transactions, 108(1), 1005-1013.

U.S. Department of Transportation, (1996). Human Factors Design Guide, Federal Aviation Administration Technical Center, United States. 